III.

\title{
Ueber die chemische Reizung von Nerven
}

nach gemeinschaftlich mit Herrn cand. med. C. Alexander angestellten Versuchen.

Von

\section{Dr. P. Grüitaner.}

Wir kommen schliesslich zu den Wirkungen der chemischen Reize. Auch hier hat man der Uebersichtlichkeit der Resultate willen wesentlich untersucht den Einfluss der verschiedenen chemischen Agentien auf motorische Nerven.

Ich gedenke in erster Linie der Arbeiten von Eckhard ') und $K$ ühne ${ }^{2}$ ), die eine grosse Reihe chemischer Substanzen prüften und sie als Nerven- resp. Nuskelreize charakterisirten. Die Reizung sensibler Nerven durch chemische Agentien war bisher nur nebenbei beobachtet und nur von Wenigen planmässig verfolgt worden.

Ich unternahm diese Untersuchungen, wie man leicht ermessen wird, in der Absicht, ebenfalls charakteristische Unterschiede in den Wirkungen ehemischer Stoffe zu beobachten, je nachdem sie auf sensible oder motorische Nerven einwirken; unsre Vermuthungen wurden aber nur zum Theil bestätigt.

Schon aus den Untersuchungen von Eckhard ${ }^{3}$ ) und namentlich von Setschenow ${ }^{4}$ ) geht hervor, dass eine Menge chemischer Agentien auf den sensiblen Nervenstamm, wie es scheint, lange nicht mit der Intensität einwirken, als auf motorische. Hängt man einen motorisehen Nerven in Kochsalzlösung, so treten alsbald Zuckungen des zugehörigen Muskels ein; thut man dasselbe mit einem sensiblen, so sieht man keine Reflexbewegung. Man be-

1) Zeitschrift für rationelle Medicin. Neue Folge Bd. I, p. 303.

2) Archiv für Anatomie etc. von Reichert u. Du Bois-Reymond. 1859. p. 213.

3) 1. c. p. 328 .

4) 1. c. p. 52 . 
merkt höchstens eine Herabsetzung der Reflexerregbarkeit fur anderweitige Reize.

Von der Richtigkeit dieser Thatsache nahmen wir mehrfach Gelegenheit uns zu überzeugen. Auch wir konnten keine ReflexBewegung an einem des Grosshirns beraubten Frosche beobachten, wenn das centrale Ende eines Hüftnerven in concentrirte Kochsalzlösung gelegt wurde. Nur ein einziges Mal sahen wir nach etwa 5 Minuten langer Einwirkung, einen Krampf auftreten, der möglicher Weise durch das Mittel hervorgerufen wurde.

Anders dagegen fallen die Resultate der Reizung mit Laugen aus. Regelmässig führt ein Frosch Reflexbewegungen aus, wenn das centrale Ende seines Hüftnerven in Natronlauge von passender Concentration gelegt wird.

Das, was wir am Frosche gesehen haben, gilt auch für die centripetalen Nerven der Sängethiere. Da es bekannt ist, dass schon ungemein schwache elektrische Ströme das centrale VagusEnde erregen und die Athmung beeinflussen können, so versuchten wir, denselben Nerven auch chemisch zu reizen. Wir legten an Kaninchen in oben beschriebener Weise Luftröhren-Fisteln an und brachten das centrale Ende eines Vagus mit der betreffenden Flüssigkeit in Berührung. Ueber 7 Minuten blieb hin und wieder der Nerv in Kochsalzlösung liegen, niemals aber zeigte sich eine Wirkung auf die Athmung. Die während dieser Zeit aufgezeichneten Athmungs-Curven waren vollständig denen gleich, welche wir beobachteten, während der Nerv nicht in der Lösung lag. Anderweitige z. B. elektrische Reize hatten anch nach längerer Einwirkung der Kochsalzlösung die bekannten Erfolge.

Vom Glycerin, welches von Kühne als intensiver Nervenreiz kennen gelehrt wurde, konnten wir in der ersten Zeit seiner Einwirkung bestimmte Effekte auf die Athmung nicht nachweisen. Dagegen machte sich jedesmal eine Verlangsamung der Athmung mit mehr oder weniger grossen Exspirations-Stillständen bemerklich, sobald der Nerv in Natron-Lauge gelegt wurde oder das Glycerin länger auf ihn einwirkte ${ }^{1}$ ).

Auch auf das centrale Ende des Ischiadicus zeigte sich nur

1) Während des Druckes dieser Arbeit erfahre ich, dass auch Langen * dorff ähnliche Untersuchungen über diesen Gegenstand mit wesentlich denselben Resultaten angestellt hat. 
die Natron-Lange, dagegen nicht die Kochsalzlösung wirksam. Steigerung des Blutdrucks trat nur im ersten Falle auf, während, was kaum wiederholt zu werden braucht, das Einlegen des peripheren Ischiadicus-Endes in Kochsalzlösung regelmässige fibrilläre Zuckungen der Pfote zu Stande brachte.

Wir haben sonach hier die eigenthïmliche Thatsache vor uns, dass chemische Stoffe, welche intensiv und binnen kurzer Zeit auf motorische Nerven einwirken, keinen Reiz ausiiben anf sensible, während die früher angewandten, sowohl die thermischen als auch die elektrischen Reize, stets die sensiblen Nerven in Erregung versetzten, nicht dagegen die motorischen.

Wenn man sich nach der Ursache jener eigenthümalichen Ausnahmestellung fragt, welche chemische Reize gegenuiber den erstgenannten 'einnehmen, so kann man eine Erklärung dafür meines Erachtens nur in der Art der Erregung der Nervenstämme finden.

Wie bekannt, geräth ein Muskel, dessen motorischer Nerv in Kochsalz eingelegt wird, zunächst nicht in Krämpfe, sondern es werden in gewissen zeitlichen Abständen verschiedene motorische Fasern erregt, so dass der Muskel in ein fibrilläres Zucken ${ }^{2}$ ) verfällt. Einlegen des Nerven dagegen in Natronlauge bedingt gewöhnlich bald auftretende, fast gleichzeitige Contraction sämmtlicher Muskelfasern oder sogar einen kurz dauernden Krampf.

Wird also ein sensibler Nervenstamm in Kochsalzlösung gelegt, so sind wir aus den Thatsachen, welche wir an der Art der auftretenden Muskelcontractionen beobachtet haben, berechtigt anzunehmen, dass auch verschiedene sensible Fasern zu. verschiedenen Zeiten erregt werden. Es kommen somit diese Reize zu verschiedenen Zeiten und an verschiedenen Stellen des Centralorgans vor; bald wird eine Ganglienzelle beziehungsweise eine ganze Gruppe von Zellen erregt, bald darauf eine zweite oder dritte, jede aber nur ein einziges Mal. Demzufolge summiren sich auch diese Reize nicht, weil sie nicht dieselben Ganglienzellen hintereinander erregen, sondern unstät ihren Ort wechseln. Würden die nämlichen Reize in gleicher Stärke und Reihenfolge in demselben Nervenstamme in das Centralorgan anlangen und dieselben cen-

1) S. auch dieses Archiv. Bd. XI, p. 195. Ueber die Höhe des Muskeltones etc. von J. Bernstein. 
tralen Apparate afficiren, so wiirden Reflexbewegungen eintreten, wie dies in der That bei verschiedenen andern Reizungen und vielleicht auch bei der durch Natronlauge der Fall ist. Denn dieselbe erregt die Nerven höchst wahrscheinlich an und für sich stärker, zweitens erregt sie sie in anderer Art. Hier geschehen die Entladungen der Reize in „Salven" 1), welche zu gleicher Zeit bestimmte Theile des Centralorgans treffen, dort beim Kochsalz nach Art des „Pelotonfeuers" ${ }^{1)}$, bei welchem die einzelnen Geschosse zu verschiedenen Zeiten und wie wir annehmen müssen, auch in verschiedenen Punkten einschlagen. Diese Art der Erregung würde somit Reflexdepression, vielleicht auch Schmerz, jene die Reflexe selber erzeugen.

Hiermit dürfte es vielleicht zusammenhängen, warum jene beiden Chemikalien so verschieden auf sensible Nervenstämme wirken und warum ferner auch schmerzhafte Erregungen so selten zu Reflexen Veranlassung geben ${ }^{2}$ ).

\section{Schlussbemerkungen.}

Versuchen wir nun am Schlusse dieser Arbeit die in ihr niedergelegten Thatsachen zusammen zu fassen und unter einen gemeinschaftlichen Gesichtspunkt zu bringen, so gelangen wir zu der Ansicht, dass es eine Menge von Nervenerregungen giebt, welche in der versehiedensten Weise auf die verschiedenen Endapparate ibertragen werden; die eine Art yon Reizen versetzt gewisse Endapparate in heftige Erregung, die andere beeinflusst sie gar nicht; die Wärme und der constante Strom reizen wesentlich centripetale, nicht centrifugale Nerven, gewisse chemische Stoffe wieder mehr die peripheren, als die centralen Apparate und unter den centrifugalen Nerven nehmen die Erweiterer der Hant eine eigenthümliche Ausnahmestellung ein.

Da wir nun keinen Grund haben anzunehmen, dass gleichartige, beispielsweise thermische Reizungsvorgänge in verschiedenen Nervenstämmen in ungleicher Weise fortgepflanzt werden, so müssen wir die Verschiedenartigkeit der Reizaffecte allein oder

1) Diese Bezeichnungen sind der interessanten soeben erschienenen Arbeit Brücke's: Ueber willkürliche und krampfhafte Zusammenziehungen der Muskeln (Sitzungsber. der k. Akademie der Wissensch.) entnommen.

2) S. dieses Archiv Bd. XVI, p. 47. Einige Versuche und Fragen etc. von $P$. Grützner und $R$. Heidenhain. 
wesentlich anf die verschiedene Erregbarkeit der nervösen Endapparate schieben. Hieraus folgt aber für die normaler Weise in den Nerven ablaufenden Reize, welche entweder vom Centrum nach der Peripherie oder in umgekehrter Richtung sich bewegen, dass auch sie aller Wahrscheinlichkeit nach untereinander weder an Intensität noch an Schnelligkeit der Aufeinanderfolge gleich sind. Es ist nicht wahrscheinlich, dáss in den motorischen Nerven dieselben Erregungswellen durch die Centralorgane erzengt werden, wie in den centripetalen durch Reizung der peripherischen nervösen Ausbreitungen.

Ein Urtheil iuber diese Angelegenheit glaubten wir gewinnen zu können, wenn wir die im Nervenstamme selbst wahrnehmbare, objective Veränderung, welche jede Reizung begleitet, die negative Schwankung seines elektrischen Stromes, studirten. Ich habe dahin gehende Untersuchungen mit Herrn Alexander bereits angestellt und die negative Schwankung beobachtet, welche durch chemische oder thermische Reizung erzeugt wird; aber die hierbei gewonnenen Resultate haben uns vorläufig noch nicht in den Stand gesetzt, ein endgiiltiges Urtheil in jener Frage zu fällen. Sie eignen sich daher noch nicht zur Veröffentlichung, dürften aber, weiter fortgesetzt, uns wohl zu dem erwünschten Ziele führen.

\section{Erklärende Bemerkungen zu der Tafel.}

Alle Curven sind auf die Hälfte reducirt; die an sie geschriebenen Zahlen bedeuten bei thermïschen Reizungen die Temperaturgrade, welche das Wasser im Wärmekästchen gerade in diesem Moment zeigte, bei elektrischen den Abstand der Inductionsrollen in Millimetern. S. bedeutet Schliessung, O. Oeffnung eines Stromes, dessen Richtung die Pfeile angeben. Alle Curven sind von rechts nach links zu lesen. 